\title{
'Rush' type retinopathy of prematurity: report of three cases
}

\author{
I NISSENKORN, I KREMER, E GILAD, S COHEN, AND I BEN-SIRA
}

From the Department of Ophthalmology, Beilinson Medical Center, Petach Tikva and the Sackler School of Medicine, Tel Aviv University, Israel

SUMMARY Three premature infants observed to develop severe stage III retinopathy of prematurity (ROP) at 3 to 5 weeks of age received immediate treatment by cryoablation and photocoagulation, with good results. The critical importance of the ophthalmic examination of premature babies from the age of 2 weeks, so as not to overlook such cases of 'rush' type ROP is stressed and the difficulty involved in treating such small neonates is discussed.

In the great majority of premature babies with retinopathy of prematurity (ROP) the disease develops gradually and relatively slowly. The early stages of ROP usually appear six to eight weeks after birth. ${ }^{1-3}$ Therefore the Committee on Fetus and Newborn of the American Academy of Pediatrics ${ }^{1}$ and Palmer ${ }^{23}$ recommended that the eyes of premature infants should be examined on discharge from the nursery or at the age of 7 to 9 weeks. Reports in the Japanese literature, however, indicate that ROP may also take a more rapid course within the first month of life. ${ }^{+6}$ These investigators found that at the age of 3 to 5 weeks the disease present in zone I or II rapidly progressed to severe ROP (stage III according to the new international classification?) and they termed this 'rush' type ROP, concluding that the prognosis for vision was very poor.

We describe here three infants with 'rush' type ROP in whom treatment by cryoablation and photocoagulation yielded good results.

\section{Case reports}

\section{CASE 1}

A female infant was born in the 26th week of gestation with a birth weight of $950 \mathrm{~g}$. The apgar score was 6 at birth and 8 five minutes later. Assisted ventilation was immediately started with $100 \%$ oxygen and continued for three weeks with $\mathrm{FiO}_{2}$ (fraction inspiratory oxygen) $25 \%$. The $\mathrm{Po}_{2}$ was maintained at $50-70 \mathrm{mmHg}$, but there were three short episodes of $\mathrm{Po}_{2}$ above $100 \mathrm{mmHg}$. The first eye

Correspondence to Dr I Nissenkorn, Department of Ophthalmology, Beilinson Medical Center, Petach Tikva, 49 100, Isracl. examination made at the age of two weeks disclosed remnants of tunica vasculosa lentis and a hazy vitreous in both eyes. The fundus examination revealed the presence of retinal vessels in zone 1 . The angle between the large vessels was $120^{\circ}$. At the age of 3 weeks congestion of posterior pole vessels and the beginning of fibrovascular proliferation (FVP) were found. At 4 weeks there was severe congestion of the iris vessels with rigidity of the pupils, and the fundus examination showed $360^{\circ}$ of severe stage III ROP. Both eyes showed similar retinal changes (Figs. 1,2) and the angle between the large vessels was $100^{\circ}$.

Under general anaesthesia a conjunctival incision 3-4 $\mathrm{mm}$ in length was made in each quadrant. After undermining with blunt-ended scissors, a cataract cryoprobe was inserted into each of the incisions well behind the equator, and cryotherapy of the avascular retina was performed through $360^{\circ}$. Particular care was taken to defrost the probe before moving it to another location. Because the inferior oblique muscles interfered with the cryopexy probe owing to the posterior location of the disease, the avascular retina close to the macula was photocoagulated with xenon light. Twenty-four hours after this procedure the iris vessels were less engorged and the pupils were less rigid. The FVP started to disappear, and three weeks later progression of the retinal blood vessels between the cryopexy scars was observed (Figs, 3 and 4). The angle between the large vessels was $70^{\circ}$, but the macula was not displaced.

Three months later the eyes appeared to be orthophoric with central fixation, and there was no nystagmus. The anterior segments were normal, and 
Fig. 1 Fundus photograph of the right eye of case I (at 30 weeks of gestation) showing the posterior pole with congested vessels in zone I only and extensive fibrovascular proliferation through $360^{\circ}$ at the edge of the advancing vasculature (severe ROP, stage III).

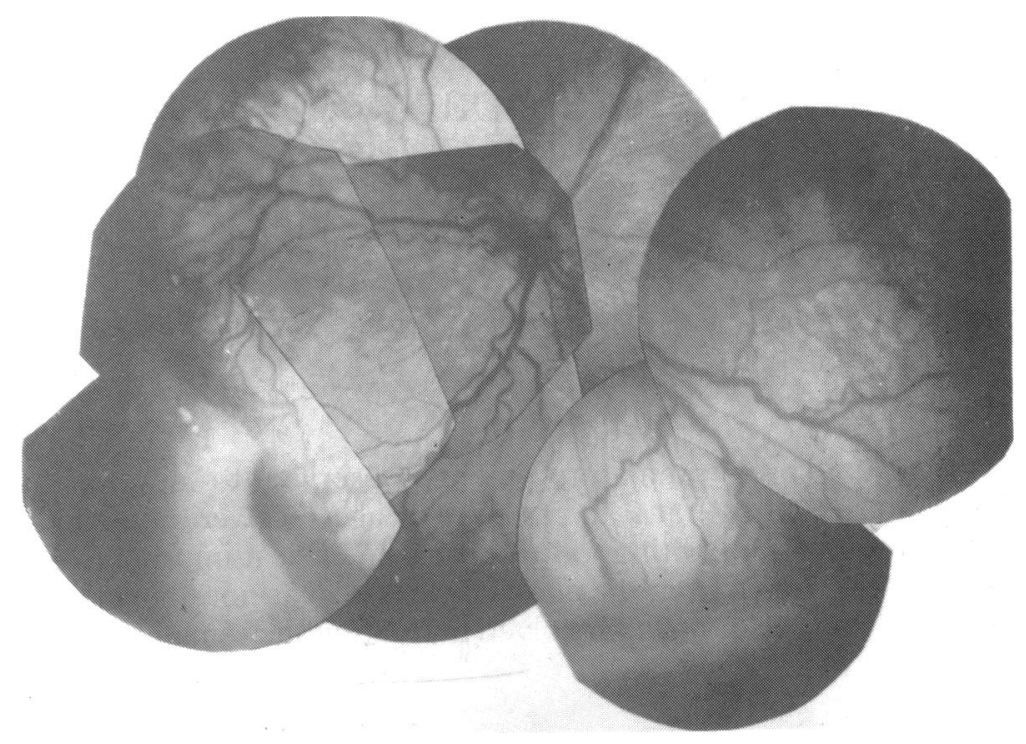

was 50 to $70 \mathrm{mmHg}$. Oxygen treatment was given for 10 days. At the age of 2 weeks examination revealed normal anterior segments in both eyes and remnants of tunica vasculosa lentis. Fundus examination showed retinal vessels only in zone $\mathrm{I}$ in both eyes. At the age of 4 weeks there were pupillary rigidity and engorgement of the iris vessels, with active stage III of severe ROP in zone I-II, $360^{\circ}$. On the same day cryotherapy was carried out on the avascular retina anterior to the fibrovascular proliferation. The procedure was performed under local anaesthesia achieved with drops of a local anaesthetic and four injections of lignocaine $2 \%$ into the subtenon space. Four conjunctival incisions at the limbus enabled the cataract cryoprobe to reach the entire avascular retina, so that xenon photocoagulation was not necessary.

Two weeks later disappearance of the pupillary rigidity as well as regression of the large retinal vessels congestion and the FVP were observed. At the age of 2 months fundus examination showed normal posterior poles and progression of the retinal vessels between the cryoscars in the equator, with cicatricial stage I. No nystagmus was observed, and the child has had a good fixation in both eyes.

\section{CASE 3}

This female infant was born at 26 weeks of gestation weighing $870 \mathrm{~g}$. The apgar score was 2 at birth and 5 after five minutes. Assisted ventilation with $100 \% \mathrm{O}_{2}$ was started because of left lung hypoventilation and atelectasis of the upper lobe of the right lung, with recurrent episodes of apnoea. $\mathrm{Po}_{2}$ was in the range of $70 \mathrm{mmHg}$. Oxygen was given for 12 days. Eye 


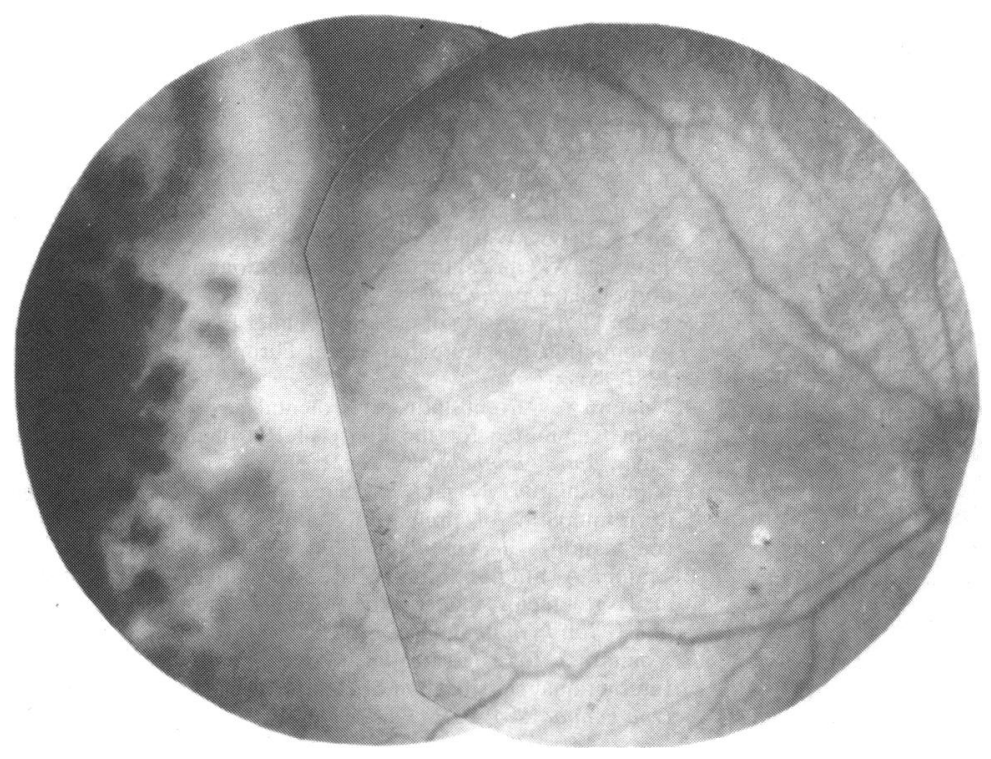

Fig. 3 Fundus photograph of the right eye of same case at the age of 3 months following

photocoagulation combined with cryotherapy. Photocoagulation and cryotherapy scars are seen temporal to the macula (bottom)

examination performed at the age of 2 weeks revealed remnants of tunica vasculosa lentis with normal anterior segments. The fundus examination showed extension of the retinal vessels to zone I. Two weeks later congestion of posterior retinal vessels was observed, and one week later, at the age of 5 weeks, severe active ROP (stage III) was found in zone I-II, $360^{\circ}$. On the same day cryotherapy was performed on the avascular retina, $360^{\circ}$, with local anaesthesia, achieved as described for case 2 . One week later the congestion of retinal vessels had lessened and there was some regression of the FVP. Three weeks later the FVP had disappeared. At the age of 3 months the angle between the large vessels

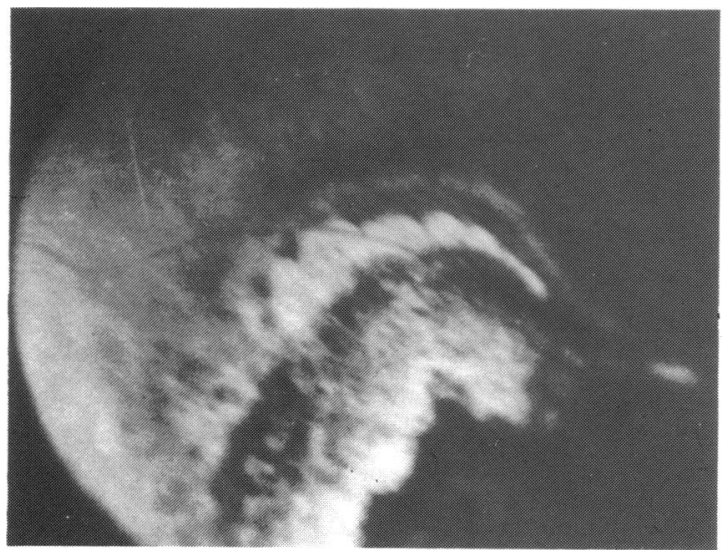

Fig. 4 Fundus photograph of the right eye of same case at the age of 3 months showing cryotherapy scars nasal to the disc, and retinal vessels progressing between the scars. was $100^{\circ}$ with some dragging on the macula. Progression of the retinal vessels between the cryoscars and towards the equator through $360^{\circ}$ was observed. The retinal changes were those of cicatricial stage II.

\section{Discussion}

The rush type ROP described in the Japanese literature $^{+.6}$ appears to occur only in premature babies of extremely low birth weight. The fibrovascular proliferative changes involve the entire circumference of the posterior retina. On the temporal side the retinal vessels reach the lateral border of the macula, and on the nasal side they run tortuously only $2-3$ disc diameters from the optic nerve head. These changes appear in zone I or II of the new international classification of ROP. ' In most cases retinal detachment was found to develop within a very short period from the onset of the retinopathic changes.

The increased chance for survival of neonates of extremely low birth weight in critical general condition creates a much larger population at risk to develop the severe stages of ROP and also rush type ROP. In our hospital the three infants presented above constitute $5 \%$ of the 50 cases of ROP requiring cryoablation treatment during recent years."

There can thus be little doubt that it would be advisable to institute routine examination of all premature infants, weighing less than $1000 \mathrm{~g}$ and born at the age of 28 weeks or less, as early as two weeks after birth. We are of the opinion that this procedure is harmless, as it is performed in the nursery intensive care unit while the baby is in the 
incubator, in which body temperature, respiration, and all the vital signs are well controlled. When the first examination is made only at the age of 7 to 9 weeks, initial retinal changes are apt to be missed, and in these eyes there may already be retinal detachment or severe cicatricial changes which will compromise the infant's vision.

Therefore, when rush type ROP is diagnosed immediate treatment is indicated. Although controlled clinical trials and long term results are still lacking, there is increasing evidence to show that cryotherapy or photocoagulation, or a combination of the two, are beneficial in treating active stage III ROP. ${ }^{+64-13}$ Circumferential ablation of the avascular peripheral retina, while getting as close to the advancing edge of the retinal vasculature as possible, has been shown to achieve retinal quiescence within hours." We believe that when the disease exists in zone I a combination of cryotherapy and photocoagulation is necessary to arrest it. This can be done only under general anaesthesia or with local subtenon injection. When signs of the disease are found in zone II, cryotherapy alone should suffice, and this is performed with local anaesthetic drops or four-quadrant injection of lignocaine.

An important point is the difficulty involved in the treatment of such small babies having a weight of less than $1000 \mathrm{~g}$ who require oxygen and whose general condition is unstable. For this reason a neonatologist should attend throughout the procedure. Furthermore, to prevent occlusion of the central retinal artery ${ }^{14}$ particular care must be taken not to press too vigorously on the eye during cryoapplication and also to observe continuously the central artery even while the cryoprobe is being defrosted.

In conclusion, we should like to stress again the critical importance of ophthalmic examination in premature infants from the age of 2 weeks, with immediate initiation of treatment when stage III $\mathrm{ROP}$ is diagnosed.

\section{References}

1 Eisenbaum AM. Timing of fundus examination for the diagnosis of retrolental fibroplasia. Retinopathy of Prematurity Conference Syllabus. Washington, DC, 1981: 318-24.

2 Palmer EA. Optical timing of examination for acute retrolental fibroplasia. Ophthalmology 1981; 88: 662-8.

3 Flynn JT. Discussion of paper by Palmer EA: Optimal timing of examination for acutc retrolental fibroplasia. Ophthalmology 1981; 88: 667-8.

4 Uemura Y. Current status of retrolental fibroplasia: report of the Joint Committec for the Study of Retrolental Fibroplasia in Japan. Jpn J Ophthalmol 1977; 21: 366-78.

5 Yamagishin N. Nagata $M$. Survey of the cicatricial stages of retinopathy of prematurity after treatment with photocoagulation. Folia Ophthalmol Jpn 1979; 30: 101-6.

6 Majima A. Studies on retinopathy of prematurity. 1. Statistical analysis of factors related to occurrence and progression in active phase. Jpn J Ophthalmol 1977; 21: 404-20.

7 Committee for Classification of Retinopathy of Prematurity. International classification of retinopathy of prematurity. Arch Ophthalmol 1984; 102: 1130-4.

8 Reese AB, King M, Owens WC. A classification of retrolental fibroplasia. Am J Ophthalmol 1953; 36: 1333-63.

9 Ben-Sira I, Nissenkorn I, Grunwald E, Yassur Y. Treatment of acute retrolental fibroplasia by cryopexy. Br J Ophthalmol 1980; 64: $758-62$

10 Topilow HW, Ackerman AL, Wang MF. The treatment of advanced retinopathy of prematurity by cryotherapy and scleral buckling surgery. Ophthalmology 1985; 92: 379-87.

11 Sasaki K. Ymashita Y, Mackawa T, Adachi T. Treatment of retinopathy of prematurity in active stage by cryocautery. Jpn J Ophthalmol 1976; 20: 384-95.

12 Hindle NW. Cryotherapy for retinopathy of prematurity to prevent retrolental fibroplasia. Can J Ophthalmol 1982; 17: $207-12$.

13 Nissenkorn I, Kremer I, Ben-Sira I, Cohen S, Garner A. A clinicopathological case of retinopathy of prematurity (ROP) treated by peripheral cryopexy. $\mathrm{Br} J$ Ophthalmol 1984; 68: $36-41$.

14 Tasman W. Zone I retinopathy of prematurity. Arch Ophthalmol 1985; 103: 1093-4.

Accepted for publication 2 September 1986. 